\title{
Transurethral Flexible Ureteroscopic Holmium Laser Lithotripsy for Treatment of Kidney and Upper Ureteral Calculi
}

\author{
Fan-Ping MENG ${ }^{1, a}$, An-Na WANG ${ }^{2}$, Ji GAO ${ }^{1, b, *}$ \\ ${ }^{1}$ Department of Urology,China-Japan Union Hospital,Jilin University,Changchun,Jilin, China \\ ${ }^{2}$ Department of Hepatology, Jilin Academy of Traditional Chinese Medicine, Changchun, Jilin, \\ China \\ aemail: 947410581@qq.com, bemail: 1217760@qq.com \\ ${ }^{*}$ Corresponding author
}

Keywords: Kidney and upper ureteral calculi, Flexible ureteroscopic, Holmium laser.

\begin{abstract}
This paper aims at studying the efficacy and safety of flexible ureteroscopic holmium laser lithotripsy for treatment of kidney and upper ureteral calculi. Methods. The duration of operation, postoperative complication, postoperative calculus clearance rate of this surgery were analyzed based on 58 patients with kidney and upper ureteral calculi treated by holmium laser under the flexible ureteroscope as clinical data (Kidney or upper ureteral calculi was diagnosed by ultrasound, CT or IVP, etc.) by using retrospective analysis. Results. The successful inserting rate of single flexible ureteroscope is $93.1 \%$ (54/58), mean lithotripsy time is $(56.1 \pm 4.8) \mathrm{min}$, and the total successful lithotripsy rate is $98.2 \%$ (57/58). Plain abdominal radiograph or CT was rechecked after one month, which indicated that the calculus clearance rate was $91.3 \%$ (53/58). All patients had no complications. Conclusion. The efficacy of holmium laser lithotripsy for treatment of kidney and upper ureteral calculi under the flexible ureteroscope was high. The operation was short and safe, with few complications and high success rate.
\end{abstract}

Clinically, the extracorporeal shock-wave lithotripsy (ESWL) was first choice in treating kidney stones and most ureteral calculi $<2 \mathrm{~cm}[1]$. Minimally invasive lithotripsy by rigid ureteroscope can be performed if upper ureteral calculi fails in vitro lithotripsy. However, the upper ureteral calculi are easy moving up to pelvis or calyx thereby leading to rigid ureteroscope unable to finish majority of lithotriptic therapies during endoluminal lithotripsy. In contrast, the flexible ureteroscope highlights its advantages because it can almost explore the calculi occurred in the whole urinary cavity. This paper studied 58 patients with kidney and upper ureteral calculi treated by holmium laser lithotripsy under the flexible ureteroscope in our hospital between June 2014 and June 2015. The efficacy is satisfied and reported as follows. 


\section{Materials and Methods}

A total of 58 patients were enrolled into this study, including 32 males and 26 females, with a mean age of $49.5 \pm 3.1$ years old. They were diagnosed as kidney and upper ureteral calculi via ultrasound, CT or IVP before operation, including 6 cases of upper ureteral calculi, 14 of pelvis calculi, 20 of renal middle and upper calyx calculi and 18 of distal calyx calculi. The calculi were unilateral containing 32 cases of single onset and 26 cases of multiple on set. The maximum diameter of the calculus is $0.88-2.03 \mathrm{~cm}$, mean $1.66 \pm 0.36 \mathrm{~cm}$. There were 32 cases of kidney calculi in situ and 20 cases of move-up kidney calculi after rigid ureteroscopic treatment.

\section{Results}

There were 54 out of 58 cases successfully inserted the ureteroscope. The rest 4 cases failed due to ureter bending and narrowing. $6 \mathrm{~F}$ ureteral stent tube was indwelt for expansion. The ureteroscope were successfully inserted for lithotripsy in two cases after 2 weeks. Among all successful ureteroscope inserting cases, apart from one case with pelvic calculi and no cases were found calculi at an included angle $<90^{\circ}$ between pelvis and subrenal calyx, the rest of calculi was discovered and smashed. After making comparisons between the kidney primary calculi and move-up kidney calculi from the upper ureteral calculi after rigid ureteroscope therapy, it is found that the differences of mean calculus finding rate, time of lithotripsy, postoperative rate and incidence of complications between two groups have no statistical significance when the calculus diameter has no statistical difference. Urinary catheter was removed within 1-2 days after operation. The time of lithotripsy was 44-89 minutes, mean 56.1 44.8 minutes. Patient was hospitalized for 3-5 days after operation, mean 4 days. CT or KUB was rechecked at Week 4 after operation. Five patients with calculus fragments $<3 \mathrm{~mm}$ were continuously given indwelling ureteral stent tube for liquid therapy and self-removing urinary calculi of which 3 cases were completely cleaned at recheck after 8 weeks (Table 1). No severe complications were found, such as ureteral perforation, degloving and active hemorrhage. Three cases experienced the low-grade fever and recovered to normal after positively anti-infection treatment.

Table 1. Calculus finding rate and lithagogue rate

\begin{tabular}{lcccc}
\hline Position of calculus & $\begin{array}{c}\text { Insert } \\
\text { ureteroscope } \\
\text { successfully }\end{array}$ & $\begin{array}{c}\text { Calculus finding } \\
\text { rate }\end{array}$ & $\begin{array}{c}\text { lithagogue rate(4 } \\
\text { weeks) }\end{array}$ & $\begin{array}{c}\text { Lithagogue } \\
\text { rate(8weeks) }\end{array}$ \\
\hline Upper ureter & $5 / 6$ & $100 \%(5 / 5)$ & $100 \%(5 / 5)$ & $100 \%(5 / 5)$ \\
Pelvis & $13 / 14$ & $100(13 / 13)$ & $92.3 \%(12 / 13)$ & $100 \%(13 / 13)$ \\
Upper and Middle calyx & $18 / 20$ & $100 \%(18 / 18)$ & $89.5 \%(17 / 19)$ & $94.7 \%(18 / 19)$ \\
Subrenal calyx & $18 / 18$ & $94.4 \%(17 / 18)$ & $87.5 \%(14 / 16)$ & $93.8 \%(15 / 16)$ \\
Total & $54 / 58$ & $98.1 \%(53 / 54)$ & $90.6 \%(48 / 53)$ & $96.2 \%(51 / 53)$ \\
\hline
\end{tabular}




\section{Discussion}

Currently, most upper ureteral calculi patients who have failed with in vitro lithotripsy will adopt the rigid ureteroscopic holmium laser lithotripsy. However, the upper ureteral calculi, especially close to the joint of pelvis and ureter, may be moved up to pelvis or calyx during the lithotripsy to escape the lithotriptic range under the rigid ureteroscope. In recent years, along with growth of materials and engineering discipline, the performance and lifespan of the flexible ureteroscope and related auxiliary equipment have been improved tremendously, so they are more suitable for general promotion [2-4]. The flexible ureteroscope can well deal with calculi that are hard to be treated by other lithotriptic means. At present, the technology of flexible ureteroscopic holmium laser lithotripsy for treatment of kidney and upper ureteral calculi has been increasingly applied clinically. The flexible ureteroscope technology was carried out at Urology of our hospital in 2014. We have the following experience after retrospective analysis of clinical data in 58 patients who were performed the flexible ureteroscopic holmium laser lithotripsy for treatment of kidney and upper ureteral calculi.

The main purpose of this paper is to study the efficacy of flexible ureteroscope for treatment of kidney and upper ureteral calculi. All patients selected were checked by ultrasound, CT or IVP, etc. before flexible ureteroscopic surgery in order to confirm the diagnosis. Six cases included were diagnosed as upper ureteral calculi, 16 of pelvis calculi, 18 of renal middle and upper calyx calculi and 18 of distal calyx calculi. All calculus diameters < $2.2 \mathrm{~cm}$. If the size of the calculus is smaller, the calculi are more easily to be smashed, and the duration is short with less possibility of ureteroscopy damages. The calculus diameter of flexible ureteroscopic lithotripsy is better $<2 \mathrm{~cm}$. If it is $>2 \mathrm{~cm}$, the duration of lithotripsy may often exceed 1 hour. However, under the circumstances of skilled technical operation, loose calculus density and various kinds of calculus removing tools, kidney calculus around 2 $\mathrm{cm}$ can also be treated by flexible ureteroscopic lithotripsy. Moreover, the impacts of hydronephrosis on operation are rather great when treating the kidney calculi because the view and vision of flexible ureteroscope are limited resulting in hardly finding direction when the hydronephrosis is moderate or severe. But high definition flexible ureteroscope can make up this defect. In addition, with regard to patients with kidney move-up calculi when using the rigid ureteroscope, if they are given the treatment by flexible ureteroscope once again, there is no difference between intervals of lithotripsy. However, in consideration of these patients belonging to re-operation, compared the duration of twice lithotripsy with direct flexible ureteroscopic therapy, the duration of lithotripsy is significantly prolonged $(\mathrm{P}<0.01)$. But the clearance of postoperative calculi and operative complications have no differences $(\mathrm{P}>0.05)$, which indicate that the second operation is safe and reliable. Therefore, the possibility of kidney move-up calculi from upper ureteral calculi shall be evaluated before operation. For those patients with upper ureteral calculi in a big possibility of calculi move-up, they could directly choose the flexible ureteroscopic lithotripsy.

We usually expand the ureters to $12-14 \mathrm{~F}$ before inserting when we perform the flexible ureteroscopic lithotripsy. The rigid ureteroscopy may be used when expanding the ureters, with a zebra guide wire indwelled. The ureteral expansion sheath is placed in along with the 
zebra guide wire. The flexible ureteroscope sheath can ensure the washing speed, keep a clear view, and avoid the excessive pressure in the pelvis. But for those patients in a small size, the ureteral expansion and sheath placement may have some difficulties. We usually use the rigid ureteroscope first and then the flexible ureteroscopic sheath with a thiner diameter during the operation in order to improve the success rate of ureteroscopy. Some documents have reported that flexible ureteroscopic sheath may not necessarily be used when carrying out the flexible ureteroscopy. However, this kind of circumstance is not fit for use of basket extraction in and out the ureters repeatedly. Four cases with failed indwelling ureteroscopic sheath due to tortuous or narrow ureters were given indwelling a 7F ureteral catheter. We successfully inserted the ureteroscope via trying not to place the ureteroscopic sheath but lead by a guide wire and a $50 \mathrm{ml}$ syringe with low-pressure perfusion at stage II operation [5].

The lithotriptic power shall be adjusted gradually. A proper power either meets needs of lithotripsy or avoids the excessive laser beam energy; strong refraction, damaged optical fiber; and reduced service life of flexible ureteroscope. We generally choose holmium laser with a parameter of 0.8-1.0 J/20-25 Hz. A nibble method shall be adopted as far as possible. The nibbling lithotripsy is a standardization format of ureteroscopic lithotripsy, which may guarantee the calculi being smashed below $3 \mathrm{~mm}$ as much as possible thereby avoiding forming large calculi and reducing the time of finding lithotripsy again, cutting down the lithotriptic time; improving lithotriptic efficacy. Generally, the calculus particles shall $<3$ $\mathrm{mm}$.

\section{Conclusion}

In option of holmium laser fibers, $365 \mu \mathrm{m}$ optical fiber may limit the range of flexible ureteroscope. Therefore, $200 \mu \mathrm{m}$ optical-fiber lithotripsy shall be chosen when carrying out the distal calyx lithotripsy. The ureters shall be expanded before placing a flexible ureteroscope and a rigid ureteroscope may be choosen for expansion under majority of circumstances. Hence, the rigid ureteroscopic holmium laser lithotripsy for treatment of upper ureteral calculi can be used in the first place. During the lithotripsy, if the conventional method of calculi moved up to the pelvis or renal calyces fails to find out the calculi, the flexible ureteroscope can be considered. This can either reduce the medical expenses or ease patients' financeial burden. However, by considering the problems of prolonged operation time and reduced level of anesthesia, the decision whether to keep an epidural catheter or chose the general anesthesia shall be made according to the possibility of calculi may be moved up to the kidneys from the upper ureteral calculi. This paper can only choose performing the stage II operation after 1-2 weeks due to without indwelling the epidural catheter because of calculi move-up when using the rigid ureteroscope to treat the upper ureteral calculi, as well as considering the level of anesthesia moving down. In short, we believe that the flexible ureteroscopic holmium laser lithotripsy for treatment of kidney and upper ureteral calculi is safe and reliable with a high success rate. 


\section{References}

[1] Miernik A,Wilhelm K ,Ardelt P,et al.Mordern urinray stone therapy:is the era of extracorporeal shock wave lithotripsy at an end[J].Urologe A .2012,51(3):372-378.

[2] Takana M,Yokota E,Toyonaga Y,et al.Stone attenuation value and cross-sectional area on computed tomography predict the success shock wave lithotripsy [J].Korean J Urol,2013,54(7);454-459.

[3] Lingman J,Mataga B,Evan A .Surgical management of upper urinary tract calculi.in.Cambell-Walsh Urology.Saunders Elsevier,Philaselphia,PA,2007:1437-1438.

[4] Gokhan Atis,Cenk Gurbuz,Ozgur Arikan,et al.Ureteroscopic management with laserlithotripsy of renal pelvic stones.[J].Endourol.2012,26(8):983-987.

[5] Takazawa R.Kitayama S ,Tsujii T.Successful outcome of flexible ureteroscopy with holmium laser lithotropsy for renal stone 2cm or greater[j].Intern J Urol,2012,19(6):998-1000. 